\title{
Pharyngo-Cutaneous fistula following drainage of a parapharyngeal abscess; a rare and challenging complication of tonsillitis
}

\author{
Seneviratne $\mathbf{R W}^{1}$, Wickramsinghe $\mathbf{A}^{2}$ \\ ${ }^{1}$ Faculty of Medicine, University of Ruhuna, Galle, Sri Lanka \\ ${ }^{2}$ Teaching Hospital, Karapitiya, Galle, Sri Lanka
}

Correspondence: Dr. Ranjana Seneviratne

e-mail:ranjanamst@yahoo.com

Bacterial Tonsillitis, if prolonged or poorly controlled is known to give rise to potentially serious complications such as Quincy's abscess, Ludwig's angina, retropharyngial abscess and parapharyngeal abscess. We discuss a rare complication, a pharyngocutaneous fistula which resulted from parapharyngeal abscess complicating tonsillitis and it's successful management.

\section{Case history}

A 50-year-old Army Officer with history of diabetes was treated for follicular tonsillitis as an outpatient at a Base Hospital with oral co-amoxyclav for one week. He was feeling systemically unwell and blood sugar control while on usual oral hypoglycaemics was poor. His condition gradually deteriorated and voice changed markedly. He became extremely unwell with dysphagia, odynophagia, trismus and high fever. The patient was admitted to a private hospital in Galle and ultrasonography detected a left parapharyngial abscess. His White Cell count was $17100 / \mathrm{mm}^{3}$ with $78 \%$ neutrophils. The drainage of the abscess was done promptly with an incision made along the anterior boarder of the middle third of the sternomastoid, under general anesthesia. This drained a large amount of pus which eventually grew Streptococcus pyogenis.

General condition of the patient improved after the surgery and his blood sugar was controlled with insulin. He was treated with a combination of broad spectrum intravenous antibiotics. However, three days later patient noticed a discharge coming through the previous surgical wound soon after he took liquids. Examination under anesthesia showed the area of left tonsil and larynx inflamed and nodular, casting the doubt of a pharyngeal carcinoma. There was a fistula of about $10 \mathrm{~mm}$ opening to the posterior part of the tonsillar fossa. Biopsy taken from the lesion, however, did not reveal the presence of any malignancy.

Patient was kept on nasogastric feeds for further 5 days. Fistula drainage was persist with the discharge of pus and saliva along the fistula tract, the wall of which included carotid sheath. The risk of a 'Carotid Blowout' and the possibility of other complications such as internal jugular vein thrombosis were considered and surgeons decided to accelerate the healing of the fistula. Feeding gastrostomy was done to bypass the troubled area. CT scan confirmed the absence of any residual collection. Nasogastric tube was removed to minimize irritation and bleeding around the internal opening. Drainage was minimal five days after gastrostomy but it took another 14 days for the wound to heal completely. Barium swallow on day 20 post-gastrostomy confirmed sealing of the fistula tract. Oral fluids were recommenced, gastrostomy tube was removed and patient was discharged three days later to his normal life following a total hospital stay of 30 days. Patient was back to his normal life 4 weeks after discharge.

\section{Discussion}

Parapharyngeal space is bound by constrictors, carotid sheath and small muscles of the neck. Infections of this space with abscess formation usually occur following tonsillitis or pharyngitis. Parapharyngeal abscess is the second most common deep neck abscess after peritonsillar abscess (1). 
It is much more common in children although adults who are immunocompromised due to conditions such as diabetes too may suffer (2). They present with swinging fever, sore throat, dysphagia, odynophagia, change in voice and patients are generally very unwell. Trismus and drooling is not uncommon. Aggressive approach is demanded as the condition is potentially fatal via many mechanisms ranging from upper airway obstruction, mediastinitis, "carotid blowout", internal jugular vein thrombosis and septic embolization (3).

Diagnosis is generally made with clinical findings supported by imaging. Ultrasound scan is valuable but CT scan can be considered the Gold Standard (3).

Smaller abscesses can be managed by intravenous antibiotics alone (2) or with added steroids and fine needle aspiration $(3,1)$. Due to the prevalence of streptococci and staphylococci in pathogenesis preferred firstline antibiotics are amoxicillin and clavulonic acid combinations (3). Significant proportion needs surgical drainage $(1,3)$. Techniques include intra-oral incision and external incision (4). Average hospital stay has been in the region of 12 days following surgical intervention and 8 days after conservative management (2).

Our patient developed oropharyngo-cutaneous fistula, an extremely rare complication of tonsillitis. The delayed presentation, inadequate treatment and poorly controlled diabetes mellitus were likely contributing factors. Early aggressive approach to the management of diabetes and intravenous antibiotics may probably have averted the situation. Pharyngocutaneous fistule are more common due to malignancies of the region and subsequent surgical procedures such as laryngectomy facilitated by radiotherapy scarred tissues. Authors were unable to find a case of pharyngocutaneous fistula following a parapharyngeal abscess in the literature review. Clinical examination, high WBC count and USS were sufficient for the diagnosis. Although parapharyngeal abscess was managed promptly with incision and drainage, irritation by subsequent nasogastric tube may have contributed to fistula formation making internal wall to break down. After identifying the internal opening under anaesthesia, this unexpected and dangerous complication was dealt by early feeding gastrostomy abandoning the widely used option of conservative measures including nasogastric feeding (5).
This intermediate procedure contributed to early recovery of the patient while minimizing the risk of fatal complications. It also avoided the need for advanced surgical options such as flaps (5). Another intermediate option would have been to introduce parenteral nutrition instead of feeding gastrostomy but it has its own share of inherent complications. Total hospital stay was prolonged to 30 days but it was unavoidable for this rare complication. Early gastric or jejunal stoma has a value in settling many infective and inflammatory complications of upper gastrointestinal tract and avoiding life threatening complications.

\section{References}

1. Oh JH, Kim Y, Kim CH. Parapharyngeal abscess: comprehensive management protocol. Orl Journal of Otorhinolaryngol Relat Spec. 2007; 69(1): 37-42. Epub 2006 Nov 2.

2. Alaani A, Griffiths H, Minhas SS et-al. Parapharyngeal abscess: diagnosis, complications and management in adults. Eur Arch Otorhinolaryngol 2005; 262 (4): 345-50. Eur Arch full text) - doi:10.1007/s00405-004-0800-6 Pubmed citation.

3. Croche Santander B, Prieto Del Prado A, Madrid Castillo MD et-al. [Retropharyngeal and parapharyngeal abscess: experience in a tertiary-care center in Seville during the last decade]. An Pediatr (Barc) 2011; 75(4): 266-72. doi:10.1016/j.anpedi.2011.03.010 - Pubmed citation.

4. Page C, Biet A, Zaatar R, Strunski V. Parapharyngeal abscess: diagnosis and treatment. ENT and Head and Neck Surgery Department, University Hospital of Amiens, Amiens, France.Cyril.page@caramail.com

5. Olasz L, Németh A, Nyárády Z. Surgical closures of oropharyngocutaneous fistulas, Plast Reconstr Surg 2000 Dec; 106(7): 1577-81. Department of Oral and Maxillofacial Surgery, Medical University Pécs, Dischka, Hungary. 